\title{
Ab initio electronic structure, optical, and magneto-optical properties of MnGaAs digital ferromagnetic heterostructures
}

\author{
Patrizia Rosa, ${ }^{1,2}$ Davide Sangalli, ${ }^{2,3,4}$ Giovanni Onida, ${ }^{1,4}$ and Alberto Debernardi ${ }^{2, *}$ \\ ${ }^{1}$ Dipartimento di Fisica, Univesità degli studi di Milano, via Celoria 16, I-20133 Milano, Italy \\ ${ }^{2}$ CNR-IMM, Unità Operativa di Supporto Agrate Brianza, via Olivetti 2, I-20864 Agrate Brianza, Italy \\ ${ }^{3}$ CNR-ISM, Unità Operativa di Supporto di Montelibretti, via Salaria Km 29.3, I-00016 Montelibretti, Italy \\ ${ }^{4}$ European Theoretical Spectroscopy Facility, Via Celoria 16, 20133 Milano, Italy
}

(Received 9 December 2014; published 24 February 2015)

\begin{abstract}
We report on a theoretical study of the electronic, optical, and magneto-optical properties of digital ferromagnetic heterostructures based on $\mathrm{Mn} \delta$-doped GaAs. We consider different structures corresponding to Mn contents within the range $12 \%-50 \%$ and we study how the system changes as a function of the doping concentration. Our first-principles approach includes the spin-orbit interaction in a fully relativistic pseudopotential scheme and the local-field effect in the description of the optical absorption. We show that $\mathrm{Mn} \delta$-doped GaAs shares many properties with the uniformly doped $\mathrm{Ga}_{1-x} \mathrm{Mn}_{x}$ As system, i.e., half-metallicity, similar absorption spectra, and moderate Kerr rotation angles in the visible spectral region.
\end{abstract}

DOI: 10.1103/PhysRevB.91.075207 PACS number(s): 71.45.Gm, 75.70.Cn, 75.70.Tj, 78.20.Ls

\section{INTRODUCTION}

Digital ferromagnetic heterostructures (DFHs) have been recently proposed as a way to overcome some of the drawbacks of random-doped $\mathrm{Ga}_{1-x} \mathrm{Mn}_{x}$ As diluted magnetic semiconductors (DMSs), without loosing the peculiar properties of the latter connected with the possibility to exploit spin-polarization effects within a semiconducting (or half-metallic) medium compatible with the present technology of semiconductor electronic devices [1]. Mn-based DFHs in III-V semiconductors are superlattice heterostructures where (fully or partially filled) monolayers (MLs) of Mn, which is substitutional to cation group III elements, are spaced by a fixed number of layers of the III-V compound. On one hand, these $\delta$-doped structures allow a concentration of $\mathrm{Mn}$ higher than in random-doped (Mn) III-V semiconductors, and thus, due to the different valence between the group III atom and the substitutional $\mathrm{Mn}$, can produce in principle a larger concentration of holes, enhancing the Curie temperature $\left(T_{C}\right)$ of the system. On the other hand, DMS $T_{C}$ can increase considerably also because it depends on the spatial distribution of magnetic ions, which can change substantially in low-dimensional systems: Nazmul and co-workers [2] showed that in a $p$-type selectively doped III-V heterostructured composed of $\mathrm{Mn} \delta$-doped GaAs/ $p$-AlGaAs a remarkably high $T_{C}$ of about $250 \mathrm{~K}$ can be reached; in Ref. [3] Chen et al. report $T_{C}$ well above room temperature in $\mathrm{GaSb} / \mathrm{Mn}$ digital alloys.

Possible applications of DMSs range from spintronics (i.e., the idea to use the spin degree of freedom in order to transmit, write, or store digital data) [4,5] to the use of spin-polarized currents for the study of surface magnetism and magnetic anisotropies in materials. In this respect, half metals, which are conductive in one spin channel and semiconducting in the other, are ideal candidates to build spin injectors. Many DMSs have indeed a half-metallic behavior [1]. Moreover, several DMSs also display intriguing magneto-optical

\footnotetext{
*alberto.debernardi@mdm.imm.cnr.it
}

coupling effects: an example is the magneto-optical Kerr effect [6] (MOKE), i.e., the polarization rotation of the light reflected by the surface of a magnetic material, which is at the basis of the operation of magneto-optical data storage media. Moreover, the MOKE can be used in the study of electronic structure in magnetic materials.

Previous studies of GaAs-based DFHs include the experimental realization, by means of low-temperature molecular beam epitaxy (MBE), of GaAs/Ga ${ }_{1-x} \mathrm{AsMn}_{x}$, separated by GaAs spacers of variable thicknesses [7], and measurements of their optical conductivity spectra in the infrared region [8].

On the theoretical point of view the optical and magnetooptical properties of Mn-doped GaAs have been studied by means of the linearized full-potential methods [9,10] (FLAPW approach) or by the Korringa-Kohn-Rostoker method [11], but only in the case of a uniform $\mathrm{Ga}_{1-x} \mathrm{Mn}_{x}$ As doping. Ab initio theoretical predictions for the magneto-optical properties of DFHs are hence lacking.

A possible advantage of $\mathrm{DFH} \delta$-doped structures with respect to uniformly doped $\mathrm{Ga}_{1-x} \mathrm{Mn}_{x}$ As DMSs derives from the possible amplification of MOKE effects by exciton confinement. However, also local field effects (corresponding indeed to the electron-hole exchange in the excitonic Hamiltonian [12]) may play an important role in such a strongly anisotropic system. However, the intrinsic complexity of DFHs, with unit cells containing many atoms, makes the direct application of full-potential methods such as those of Refs. [9,10] computationally prohibitive.

On the other hand, more efficient $a b$ initio methods based on pseudopotentials (PPs) and plane waves have not been systematically used for the study of MOKE spectra, because of the common wisdom that an all-electron approach was required in order to describe the wave function within the core region [where the spin-orbit ( $\mathrm{SO}$ ) interaction is stronger]. Instead, some of us have recently demonstrated that, provided that the spin-orbit interaction is fully taken into account in the construction and use of "fully relativistic" PPs, the planewave-based approach provides results of the same quality as those of all-electron calculations [13]. 
Indeed the MOKE is due to a different dielectric response for right $\left(\epsilon_{+}\right)$and left $\left(\epsilon_{-}\right)$circularly polarized light, which stems from the difference between the wave functions with $+L_{z}$ character and $-L_{z}$ character. In a magnetic material this difference is due to the SO coupling in the Hamiltonian, $H=\xi \mathbf{L} \cdot \mathbf{S}$, which transfers the spontaneous symmetry breaking between the $+S_{z}$ and the $-S_{z}$ spin component to the spatial part of the wave function. Thus the MOKE cannot be captured by a perturbative description of the SO effect or by the use of scalar relativistic PPs, which only include the effect of the SO in the energy levels without affecting the wave function.

In this work we hence perform first-principles simulation of $\mathrm{Mn} \delta$-doped GaAs structures [7], including the effect of the SO in a nonperturbative way and using fully relativistic PPs. We then compute the absorption coefficients of the system and the MOKE parameters in the so-called polar geometry, where the magnetization vector is oriented perpendicular to the reflective surface and parallel to the plane of incidence. This is the most studied geometry and the one which gives the largest Kerr rotation.

This work is organized as follows. In Sec. II we present DFT-LDA ground state results and Kohn-Sham (KS) band structure for heterostructures described by 8-, 6-, 4-, and 2-layer supercells (SCs), corresponding to doping concentrations from $12.5 \%$ to $50 \%$ (see Fig. 1). We show the electronic density of states, projected onto spin components, atomic species, and $s / p / d$ angular momenta. In Sec. III we deal with optical absorption spectra, computed within the linear response scheme in the random-phase approximation (RPA), and compared with experiments. We then extend our analysis to magneto-optical effects in Sec. III A, after briefly
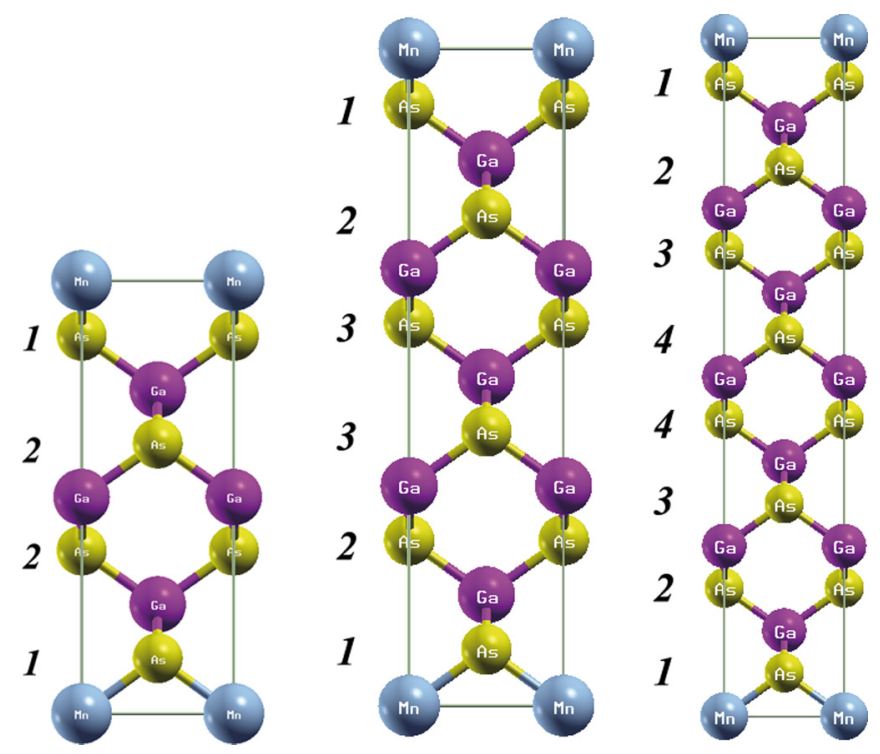

FIG. 1. (Color online) Atomic structure (unit cells) of some of the studied $\delta$-doped materials. Yellow spheres are As atoms, the violet ones represent $\mathrm{Ga}$, and the cyan color is used for $\mathrm{Mn}$. The corresponding Mn-doping concentrations are $12.5 \%$ (rightmost structure, an 8-layer supercell with $7 \mathrm{GaAs}$ layers separating MnAs ones); $16.7 \%$ (6-layer supercell with $5 \mathrm{GaAs}$ layers); $25 \%$ (leftmost, 4-layer supercell with $3 \mathrm{GaAs}$ layers); 50\% (2-layer supercell, not shown). summarizing the basic equations for the MOKE and MCD spectra, and we discuss our results analyzing the similarities and differences with the case of uniform doping. Finally, in Sec. IV we draw our conclusions.

\section{ELECTRONIC PROPERTIES OF Mn-DOPED GaAs: $\delta$ DOPING}

We model Mn $\delta$-doped GaAs by means of the periodic superstructures shown in Fig. 1. The variable thickness of bare GaAs interposed between MnAs layers allows us to simulate $\delta$-doped materials with $12 \%, 16 \%, 25 \%$, and $50 \%$ of Mn atoms replacing $\mathrm{Ga}$ ones (with the latter case corresponding to a hypothetical GaAs/MnAs superlattice).

We perform our calculations with DFT-LDA [14-16] in the plane-wave PP scheme, as implemented in the Quantum ESPRESSO (QE) package [17]. To take into account the spin-orbit effects on the valence wave functions, the use of fully relativistic (norm-conserving) PPs is necessary. Moreover, the presence of noncollinear spin polarization (i.e., spinorial KS wave functions) must be allowed. Both features are available in QE. We use tetragonal unit cells, by relaxing the lattice parameter along the growth direction (perpendicular to the Mn-doped layers) and all the atomic positions within the unit cell [18]. The in-plane lattice parameter is kept fixed at its bulk $\mathrm{GaAs}$ value, consistently with an epitaxial growth on the GaAs (001) oriented substrate. Ground-state calculations are performed with a kinetic energy cutoff of $50 \mathrm{Ry}(680 \mathrm{eV})$ for the wave functions, and a Monkhorst-Pack [19] grid $12 \times 12 \times n$ for the Brillouin zone (BZ) with $n=8,4,3,2$ for the two-, four-, six-, and eight-layer SCs, respectively, thus maintaining an (approximately) uniform mesh in the BZ. The ground state of

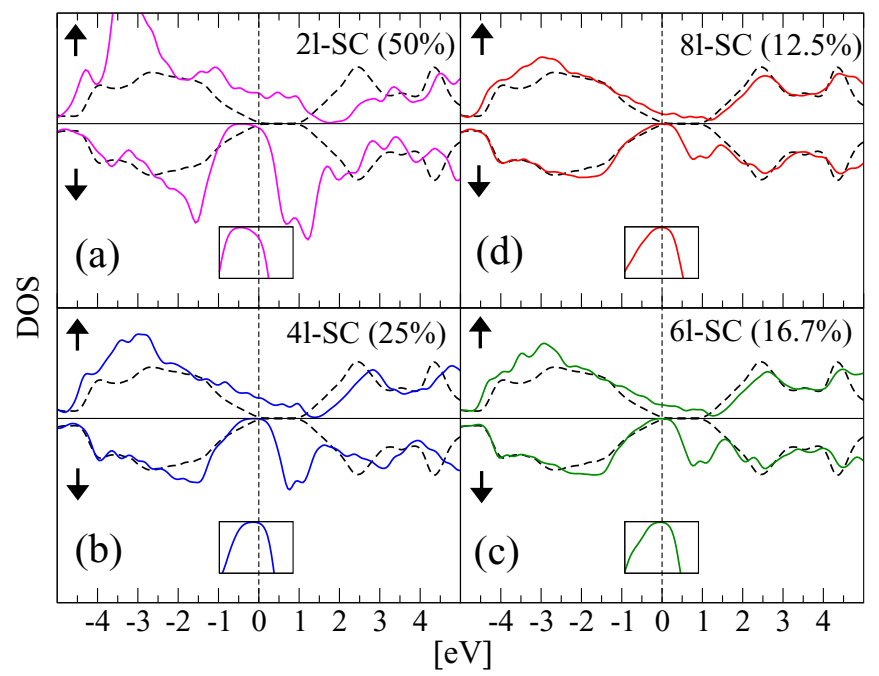

FIG. 2. (Color online) Spin-resolved density of states of the four studied Mn-doped GaAs heterostructures, compared with that of bulk GaAs (black dashed line). Panels (a) to (d) correspond to decreasing Mn concentrations, from $50 \%$ to $12.5 \%$. Spin-up and spin-down components are computed by projecting the spinorial wave functions onto the $S_{z}$-spin eigenstates. The inset of each figure shows a zoom in the vertical scale of the density of states in the minority-spin channel close to the Fermi level. 


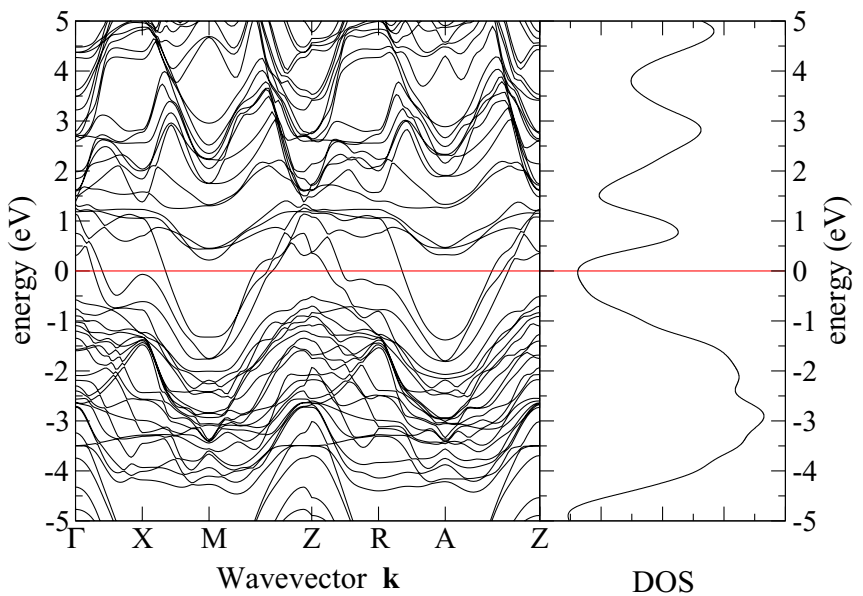

FIG. 3. (Color online) Kohn-Sham band structure and total DOS for the 25\% doping case [panel (b) of Fig. 2]. The red line is drawn at the Fermi energy.

bulk GaAs, which is also considered for reference, is computed using a $12 \times 12 \times 12$ sampling.

We compare the density of states at the KS level [20], for the four systems considered, in Fig. 2. All the Mn-containing systems have a metallic character, as expected. The case of undoped bulk GaAs is also shown for comparison. Doped systems clearly display a nonzero DOS at the Fermi level, with a characteristic Mn-related structure lying in the top region of the bulk band gap. The 50\% doped structure [panel (a)] clearly deviates from the behaviors shown by lower-doping systems, both at the level of the DOS and in term of Fermi surface topology. This difference can be clearly seen in the band structure, where the number of bands appearing to cross the Fermi level increases from three (see, e.g., Fig. 3), for the lower doping cases, to four (see Fig. 4) in the 50\% doped system. At the same time, the DOS of the $50 \%$ doped case displays a clear reduction of the spectral weight around $2.5 \mathrm{eV}$ above $E_{F}$, typical of bulk GaAs, in favor of lower energy structures.

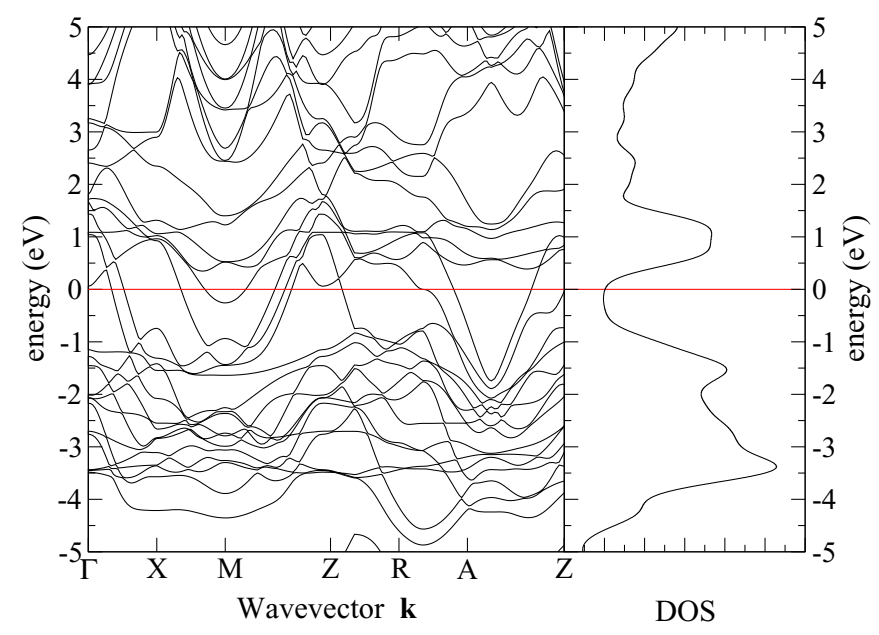

FIG. 4. (Color online) Kohn-Sham band structure and total DOS for the 50\% doping case [panel (a) of Fig. 2]. The red line is drawn at the Fermi energy.
Moreover, the total magnetic moment per unit cell is an integer multiple of $\mu_{B}$ (namely, $4 \mu_{B}$ ) in all the structures except the $50 \%$ doped one, where it drops slightly below the value of $4 \mu_{B}$. A noninteger value of $\mu / \mu_{B}$ implies that the system cannot be a half metal, as also appearing from spinprojected DOS (Fig. 2), where both the spin-up and spin-down component of the corresponding DOS are nonnegligible at the Fermi energy. In the inset of each panel we display the spindown component of the DOS, zoomed in the vertical scale.

The $12 \%, 17 \%$, and $25 \%$ DFHs present a gap in the spindown projected DOS, which is zero at the Fermi energy. At variance, the spin-up DOS of all these DFHs is clearly nonzero at the Fermi energy. Hence, the electron population at the Fermi energy in the $12 \%, 17 \%$, and $25 \%$ DFHs is fully spin-up polarized, and these systems are half metals, similarly to the case of uniformly Mn-doped GaAs compounds [1].

In view of the qualitative difference of the 50\% doped system, in the following discussion and in optical property calculations only materials with integer $\mu / \mu_{B}$ will be considered, and compared with the uniformly doped systems.

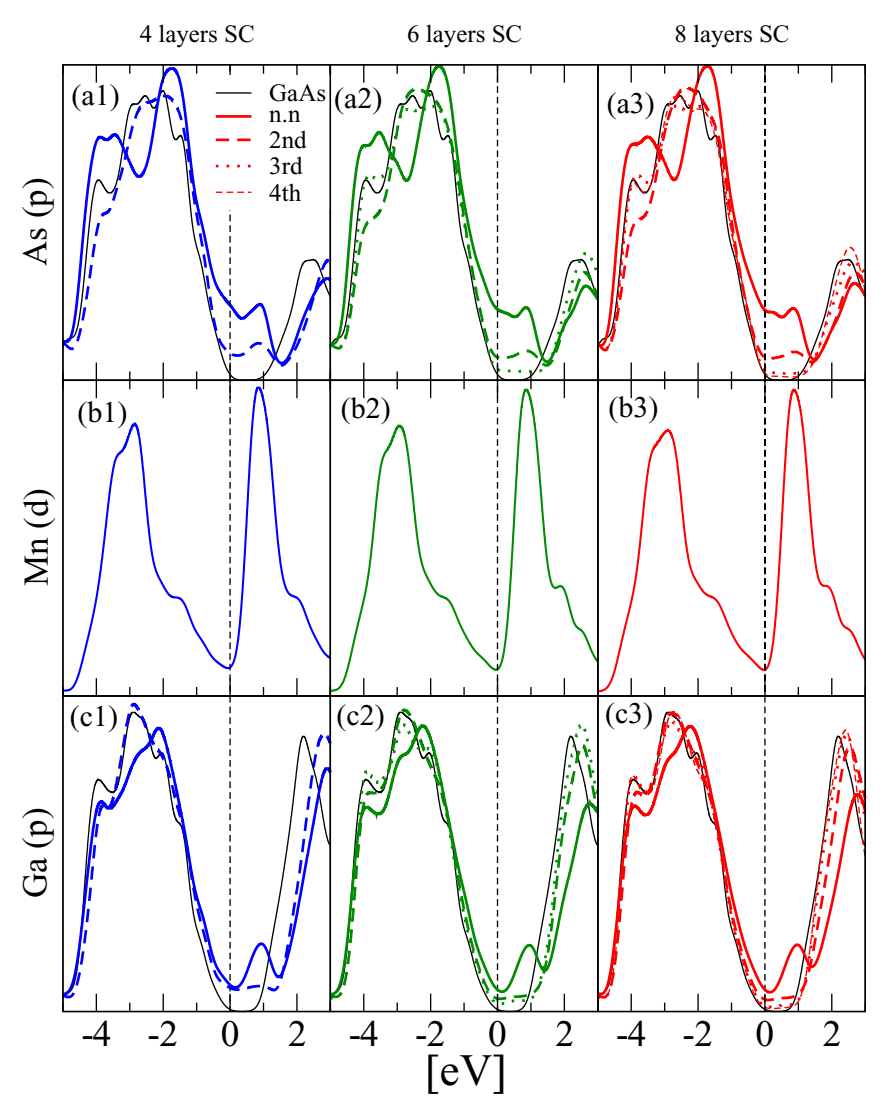

FIG. 5. (Color online) Projected density of states of the studied MnGaAs heterostructures. The projections on As $p$ orbitals [panels (a1)-(a3)], Mn $d$ orbitals [panels (b1)-(b3)], and Ga $p$ orbitals [panels (c1)-(c3)] are shown for the structures with 3 (blue), 5 (green), and 7 (red) GaAs layers between the Mn layers, respectively. The projection on As and Ga species is atom resolved. Atoms are selected according to their distance from the Mn layer, as 1st nearest neighbor (thick continuous line), 2nd (thick dashed line), 3rd (dotted line), and 4th (thin dashed line). The case of bulk GaAs is also shown for comparison (thin continuous black line). 
A more detailed analysis of the band character can be performed by decomposing the DOS via a projection on the different atomic species and angular momenta. The major contribution to the metallic bands comes from $\mathrm{Mn} d$ orbitals and As and Ga $p$ orbitals. Figure 5 shows the As $p$ (top panel), Mn $d$ (middle panel), and Ga $p$ (bottom panel) contribution for the three systems considered (i.e., three-, five-, and seven-layer supercell). The As and Ga $p$ contribution to the undoped GaAs DOS is also shown for comparison (black line). The $p$ As and Ga contributions are also decomposed according to the distance of the considered atom from the Mn layer, with the thick continuous line corresponding to the As (Ga) nearest to Mn. As shown in Fig. 5, the presence of Mn mainly affects the first two closest layers of As (labeled with 1 and 2 in Fig. 1, thick continuous and dashed lines in Fig. 5) and the first closest layer of $\mathrm{Ga}$ (same conventions). They all display a peak above the Fermi energy at the same energy of the $d$ orbital of Mn. These peaks indicate a hybridization of $d$ orbitals of Mn with $p$ orbitals of the As and Ga atoms, or better with the $s p^{3}$ backbone of the GaAs lattice. Indeed the $\mathrm{Mn}$ atoms, replacing Ga atoms, are "forced" to create an $s p^{3}$ hybridization. However, while Ga has three electrons in the $s$ plus $p$ orbitals, Mn only has two. There is then a competition between filling the hole in the $s p^{3}$ backbone and keeping five valence $\mathrm{Mn}$ electrons in its $d$ orbitals. This competition is represented as two resonant configurations in Fig. 6. The dashed red arrow indicates the electron shared between $s p^{3}$ and $d$ orbitals. In both configurations the magnetic moment is $4 \mu_{B} /$ cell.

The remaining As and Ga atoms show a PDOS contribution very similar to the one in bulk GaAs, confirming that they are not substantially influenced by the presence of the Mn dopant. This result confirms that the ferromagnetic interaction between the Mn layers becomes much weaker if they are separated by

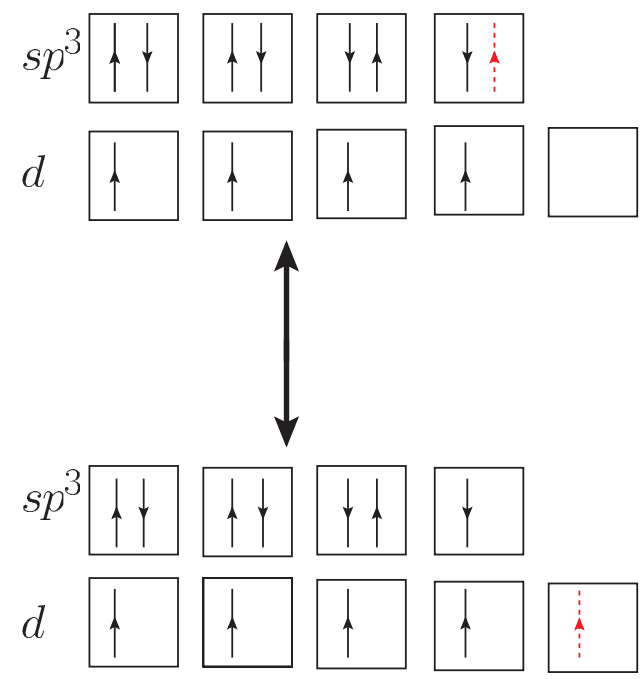

FIG. 6. (Color online) Schematic electronic diagram for the AsMn binding represented as two resonant configurations. Each Mn orbital has four first-nearest-neighbor As atoms. In a first approximation, a hole is shared between the four Mn-As $s p^{3}$ bond orbitals and the Mn $d$ orbitals. In practice the hole delocalizes also on the other $s p^{3}$ orbitals of the doped GaAs and is responsible of the ferromagnetic interaction between different Mn layers. more than $3 \mathrm{GaAs}$ layers. However also at greater distance a small projection of the state immediately above the Fermi level remains on the As and Ga orbitals, suggesting that a smaller ferromagnetic interaction is still present also in the $12 \%$ doped system, where the Mn layers are separated by $7 \mathrm{GaAs}$ layers.

\section{OPTICAL PROPERTIES OF Mn-DOPED GaAs}

Optical absorption is proportional to the imaginary part of the macroscopic dielectric function, $\epsilon_{M}(\omega)$. The latter can be obtained from microscopic quantities such as the response function $\chi_{G, G^{\prime}}(\boldsymbol{q}, \omega)[12,21]$. Response functions can in turn be computed on the basis of transition matrix elements between electronic states (both interband and intraband in the case of metals). Several levels of theory can be adopted, ranging from the simplest approach, i.e., the bare independent particlerandom phase approximation (IP-RPA), to more complete approaches where, e.g., local fields and/or excitonic effects are taken into account. In the long-wavelength limit, which is appropriate for UV-VIS spectra, $\epsilon_{M}$ can be expressed as

$$
\epsilon_{M}(\omega)=1-\lim _{\boldsymbol{q} \rightarrow 0} \frac{4 \pi e^{2}}{|\boldsymbol{q}|^{2}} \chi(\boldsymbol{q}, \omega),
$$

involving only the $G=0, G^{\prime}=0$ "head" of the appropriate response function $\chi_{G, G^{\prime}}(\boldsymbol{q}, \omega)$ written in reciprocal space [12].

In this work, we use $\chi_{G G^{\prime}}^{\mathrm{RPA}}(\boldsymbol{q}, \omega)$, i.e., the response function constructed including the local-field (LF) effects:

$$
\begin{aligned}
\chi_{\boldsymbol{G} \boldsymbol{G}^{\prime}}^{\mathrm{RPA}}(\boldsymbol{q}, \omega)= & \chi_{\boldsymbol{G}^{\prime}}^{\mathrm{QP}}(\boldsymbol{q}, \omega) \\
& +\sum_{\boldsymbol{G}_{1} \boldsymbol{G}_{2}} \chi_{\boldsymbol{G} \boldsymbol{G}_{1}}^{\mathrm{QP}}(\boldsymbol{q}, \omega) f_{\boldsymbol{G}_{1} \boldsymbol{G}_{2}}^{H}(\boldsymbol{q}, \omega) \chi_{\boldsymbol{G}_{2} \boldsymbol{G}^{\prime}}^{\mathrm{RPA}}(\boldsymbol{q}, \omega) .
\end{aligned}
$$

Here $f_{\boldsymbol{G}_{1} \boldsymbol{G}_{2}}^{H}(\boldsymbol{q} \omega)=\delta v_{H}[\rho] / \delta \rho$, the functional derivative of the Hartree potential, is the bare Coulomb interaction and corresponds to an electron-hole exchange term [12]. Neglecting $f^{H}$ one has $\chi_{\boldsymbol{G} \boldsymbol{G}^{\prime}}(\boldsymbol{q}, \omega)=\left[\chi_{0}^{\mathrm{QP}}\right]_{\boldsymbol{G} \boldsymbol{G}^{\prime}}(\boldsymbol{q}, \omega)$, i.e., the response function in the IP-RPA approximation.

The quasiparticle (QP) response function an be expressed in terms of single-particle electronic eigenstates $\left|\psi_{n k}^{\mathrm{QP}}\right\rangle$ and eigenenergies $\epsilon_{n \boldsymbol{k}}^{\mathrm{QP}}$ as

$$
\begin{aligned}
\chi_{\boldsymbol{G} \boldsymbol{G}^{\prime}}^{\mathrm{QP}}(\boldsymbol{q}, \omega) \\
=-\frac{1}{V} \sum_{n \boldsymbol{k}} \sum_{m \boldsymbol{k}^{\prime}}\left(f_{n \boldsymbol{k}}-f_{m \boldsymbol{k}^{\prime}}\right) \\
\quad \times\left(\frac{\left\langle\psi_{m \boldsymbol{k}^{\prime}}^{\mathrm{QP}}\left|e^{i(\boldsymbol{q}+\boldsymbol{G}) \boldsymbol{r}}\right| \psi_{n \boldsymbol{k}}^{\mathrm{QP}}\right\rangle\left\langle\psi_{n \boldsymbol{k}}^{\mathrm{QP}}\left|e^{-i\left(\boldsymbol{q}+\boldsymbol{G}^{\prime}\right) \boldsymbol{r}^{\prime}}\right| \psi_{m \boldsymbol{k}^{\prime}}^{\mathrm{QP}}\right\rangle}{\epsilon_{m \boldsymbol{k}^{\prime}}^{\mathrm{QP}}-\epsilon_{n \boldsymbol{k}}^{\mathrm{QP}}-\omega-i \eta}\right) .
\end{aligned}
$$

$\epsilon^{\mathrm{QP}}$ are obtained from KS eigenvalues applying a gapopening/band-stretching correction which simulates the selfenergy effects in semiconductors/metals, and $\left|\psi_{n \boldsymbol{k}}^{\mathrm{QP}}\right\rangle$ are taken as unperturbed KS eigenstates $\left|\psi_{n \boldsymbol{k}}^{\mathrm{KS}}\right\rangle$, as implemented in the YAMBO [22] code.

Here we report the optical properties of our four-, six-, and eight-layer SCs of Mn-doped GaAs. The absorption spectra of the undoped system is also shown for reference as for the case of the DOS. To obtain converged spectra a $k$-points sampling of 
the BZ with a $24 \times 24 \times n$ mesh is used, with $n=8,6,4$ for the four, six, and eight layers, respectively, considering $n v$ valence bands and $n c$ conduction bands in total. The Drude term is modeled with a plasma frequency $\omega_{p}=2.75 \mathrm{eV}$ and relaxation frequency of $0.7 \mathrm{eV}$ and the QP corrections are included as a $10 \%$ stretching of the band structure, as in Ref. [10]. LF effects are found to converge including $\boldsymbol{G}$ vectors in Eq. (2) up to a kinetic energy cutoff of 4 Ry. The absorption of bulk GaAs is instead computed with a double-grid sampling of the BZ [23] with a regular $12 \times 12 \times 12$ mesh shifted from $\Gamma$ plus a random mesh of $2000 \mathrm{k}$ points. In this case the well-established value [24] of $0.8 \mathrm{eV}$ opening of the band gap has been used for the QP correction.

In Figs. 7 and 8 the absorption spectra, $\operatorname{Im}\left[\epsilon_{M}(\omega)\right]$, for the $12 \%, 17 \%$, and $25 \%$ doped materials are shown. The case of bulk (undoped) GaAs is in panel (a) of both figures. Dashed lines show the IP-RPA spectra for comparison. The RPA spectra including LF effects differ from IP-RPA ones because the intensity of the absorption is reduced. The intensity reduction is peak specific, and is due to the well-known depolarization effect, which is usually more important in nonuniform systems. However, LF do not change substantially the position of the peaks.

We first consider incident light with polarization on the layers plane (Fig. 7). The intensity of the main E2 peak around $5 \mathrm{eV}$ is lower in DFHs than in bulk GaAs, and it decreases by increasing the Mn concentration. In fact this peak becomes

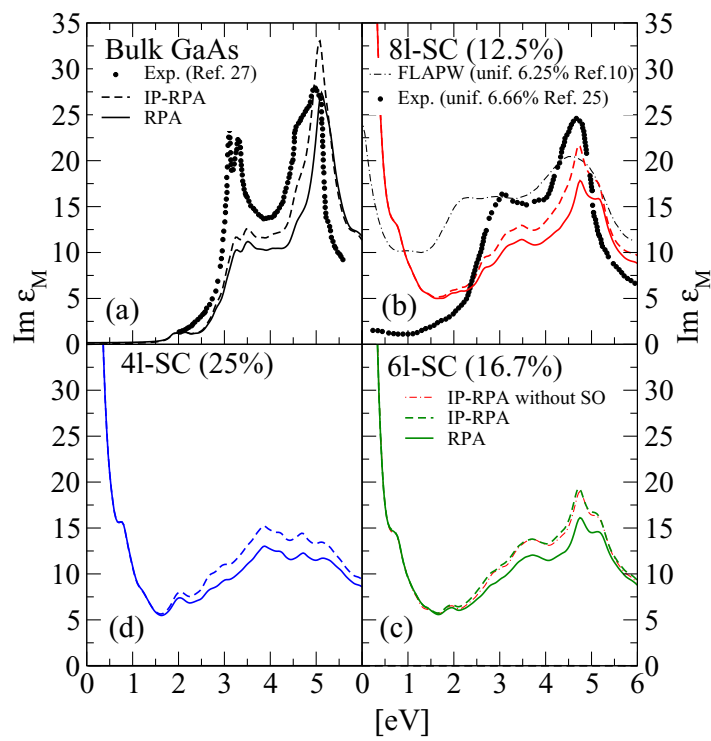

FIG. 7. (Color online) Computed UV-VIS absorption spectra for light polarization parallel to the Mn layers. Panel (a) shows the bulk GaAs case as reference, and $\mathrm{Mn} \delta$-doped heterostructures with $12.5 \%$, $16.7 \%$, and $25 \% \mathrm{Mn}$ concentration are shown in panels (b), (c), and (d), respectively. The dashed lines and continuous lines represent the IP-RPA and RPA results, respectively. Theoretical spectra are compared with experimental data for bulk GaAs (circles in panel (a), from Ref. [27]) and for a uniformly Mn-doped GaAs sample at low doping concentrations (dots in panel (b), from Ref. [25]). We also compare with the FLAPW ab initio results of Ref. [10], computed for a uniformly doped system with a concentration of $6.25 \%$ (dot-dashed line).

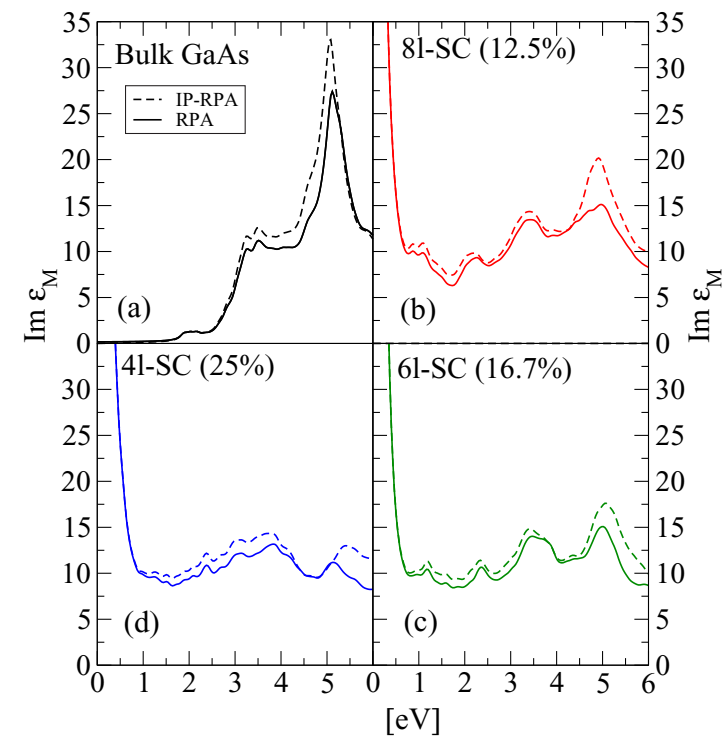

FIG. 8. (Color online) Computed UV-VIS absorption spectra of bulk GaAs [panel (a)] and Mn $\delta$-doped heterostructures with $12.5 \%$ [panel (b)], 16.7\% [panel (c)], and 25\% [panel (d)] Mn doping, for light polarization perpendicular to the Mn layers (grazing incidence). As in Fig. 7, the dashed lines and continuous lines represent IP-RPA and RPA results, respectively.

a double peak by effect of the Mn $\delta$ doping [panel (b)], and more structures appear by decreasing the distance between $\mathrm{Mn}$ layers [panels (b), (c), and (d)]. We compare in panel (b) our IP-RPA results for the $12 \% \delta$-doped systems (seven layers of Ga between the Mn layers) with experimental data [circles in panel (b)] for a uniformly doped $\mathrm{Ga}_{1-x} \mathrm{Mn}_{x}$ As sample with $x=0.066$ (Ref. [25]). The theoretical underestimation of the intensity of the $5 \mathrm{eV}$ (E2) peak is likely due to the different doping concentration between theory and experiment. Indeed the intensity of the E2 peak decreases increasing the dopant concentration, both experimentally (Refs. [25,26]) and theoretically (present work, and Ref. [10]). We notice that the double-peak structure at $\sim 5 \mathrm{eV}$ of Fig. 7 is peculiar to the $\delta$-doped GaAs absorption spectra in polar geometry, since it is absent in the computed spectra with light polarization perpendicular to the Mn layer (Fig. 8), as well as in the experimental data for randomly distributed Mn doping. The double peak also appears when the absorption spectra are computed without SO as shown in Fig. 7(c2).

The influence of local field effects in the case of grazing incidence (Fig. 8) is more pronounced for energies above $5 \mathrm{eV}$, where LFs appear to suppress the peak intensity more than at lower energies. On the other hand, in both Figs. 7 and 8 the intensity of the theoretical $\sim 3 \mathrm{eV}$ is underestimated, due to our neglect of excitonic effects [24,27]. The latter, however, are expected to be strongly suppressed in systems with efficient freecarrier screening, such as in metals or even half metals, with respect to the case of a pure semiconductor. In fact the peak at $3 \mathrm{eV}$ in the experimental data for uniform Mn doping [dots in panel (b) of Fig. 7] is clearly less prominent than in bulk GaAs.

The overestimation of $\epsilon_{M}$ at very low energy in our simulation can be attributed, at least in part, to an overestimation of intraband transitions, described by the Drude term. The 
discrepancies between experimental data and our results can also be partially accounted for by the different types of doping (uniform vs $\delta$-doping).

Moreover experiments are performed at diluted Mn concentration (a few percent or less). When the concentration of Mn in uniformly doped samples is sufficiently low, the overlap of impurity wave functions is negligible and the compound is semiconducting. At variance, in $\delta$-doped samples, the conductivity in the Mn layers remains metallic also at overall low doping concentrations.

\section{A. Kerr effect and reflection magneto-optical dichroism}

MOKE experiments evaluate the difference in optical reflectivity for left and right circularly polarized light. In practice, since a linearly polarized light can be thought of as the result of the superposition of left and right circularly polarized waves having the same intensity and phase, a nonzero difference in the dielectric response of the material can be detected as a change in the polarization properties of the reflected light.

In this way, Kerr parameters are defined in terms of the Kerr rotation $\theta_{K}$ and the Kerr ellipticity $\eta_{K}$, the first describing the angle between the major axis of the ellipse with respect to the original linear polarization direction, and the second one being related to the ratio between the two ellipse axis.

In the present paper we consider the MOKE in the so-called polar geometry, where the light propagates along the direction of the magnetization of the sample, which we identify here as $z$, perpendicular to the sample surface. The polarization hence lies in the $x y$ plane. We now introduce the complex refractive index $n_{ \pm}=\sqrt{\epsilon_{ \pm}}$, where $\epsilon_{+}\left(\epsilon_{-}\right)$is the dielectric function for the right (left) circularly polarized light. Then following Ref. [6] the Kerr parameters are defined as

$$
\theta_{K}(\omega)+i \eta_{K}(\omega)=i \frac{n_{+}(\omega)-n_{-}(\omega)}{n_{+}(\omega) n_{-}(\omega)-1} .
$$

$\epsilon_{ \pm}$can be derived, for a cubic system, from the $x x$ and $x y$ elements of the dielectric tensor, according to the expression $\epsilon_{ \pm}=\epsilon_{x x} \pm i \epsilon_{x y}$. The magneto-optical response can hence be computed starting from the diagonal and off-diagonal components of the dielectric tensor. Indeed, if $\epsilon_{x y} \ll \epsilon_{x x}$, which is the common case, Eq. (4) is well approximated by

$$
\theta_{K}(\omega)+i \eta_{K}(\omega) \simeq \frac{-\epsilon_{x y}(\omega)}{\left[\epsilon_{x x}(\omega)-1\right] \sqrt{\epsilon_{x x}(\omega)}},
$$

which holds for small Kerr angles.

Remarkably, in such differential measurements, the contribution stemming from the off-diagonal elements of the dielectric tensor, which is usually negligible in bare absorption or reflectivity experiments, becomes important. $\epsilon_{x y}$ can be obtained from the response function $\chi\left(\boldsymbol{q}_{\alpha} \boldsymbol{q}_{\beta} \omega\right)$, by generalizing Eq. (1). In practice, we evaluate the dielectric tensor by means of the YAMBO [22] code, using the same approach described in Ref. [13], where $\epsilon_{\alpha \beta}$ is computed, at the RPA-IP level, as

$$
\epsilon_{M \alpha \beta}(\omega)=1-\lim _{\substack{\boldsymbol{q}_{\beta} \rightarrow 0 \\ \boldsymbol{q}_{\alpha} \rightarrow 0}} \frac{4 \pi e^{2}}{q^{2}} \chi\left(\boldsymbol{q}_{\alpha} \boldsymbol{q}_{\beta} \omega\right) .
$$

$\epsilon_{x y}$ must be converged against the same parameters needed to converge the absorption. Convergence in the sampling of the BZ requires a $24 \times 24 \times n$ grid where the number of $k$ points along the $z$ direction are $n=12,8,6$ for the 4-, 6-, and 8-layer supercell, respectively, i.e., a slightly finer sampling along the $z$ direction, if compared to the one used for absorption. The reason stems from the fact that $\epsilon_{x y}$ is two orders of magnitude smaller than $\epsilon_{x x}$. The knowledge of $\epsilon_{\alpha \beta}$ also allows one to compute the reflectance $R_{ \pm}(\omega)$ at normal incidence, defined as the square modulus of the complex reflectivity, both for right $(+)$ and left $(-)$ circularly polarized light [6]:

$$
R_{ \pm}=\left\|\frac{n_{ \pm}(\omega)-1}{n_{ \pm}(\omega)+1}\right\|^{2} .
$$

Following Ref. [6], the latter quantities determine the reflectance magnetic circular dichroism (R-MCD) spectrum as

$$
R_{\mathrm{MCD}}(\omega)=\frac{1}{2} \frac{R_{+}-R_{-}}{R_{+}+R_{-}},
$$

which is more easily measured experimentally than the MOKE parameters. In the limit of small Kerr angles one can prove, after some tedious but straightfoward algebra, that $R_{\mathrm{MCD}}(\omega)$ and Kerr ellipticity are brought to coincide:

$$
R_{\mathrm{MCD}}(\omega) \approx \eta_{K}(\omega) .
$$

In Fig. 9 we plot the computed Kerr parameters $\theta_{K}$ and $\eta_{K}$ for the considered DFHs. We also show, by comparison, some theoretical and experimental literature data for uniformly doped MnGaAs.

We first observe that there is no clear dependence of the computed Kerr parameters on the Mn-doping concentration. This finding is in agreement with previous calculations for the uniform-doping case $[9,10]$. Our calculations show that, as it happens for the absorption, the computed MOKE is similar to the one obtained for the uniformly doped case. In Fig. 9 (bottom panels) the Kerr rotation is compared with all-electron calculations for the uniform system at the LDA level [10]. Despite this difference in the calculations, different structures (uniform doping and DFH), and different doping concentrations, all calculations and experimental data show at least one common dominant feature, that is, a negative Kerr rotation at low energy. In the experimental data for uniform doping [28,29] this negative peak in the Kerr rotation is blueshifted by $\approx 0.6 \mathrm{eV}$ with respect to our results (see Fig. 9, top left panel). This shift also appears in the computed Kerr ellipticity/R-MCD data (top right panel) and in DFT calculations for the uniform-doping case. It maybe due to inadequacy of the $10 \%$ band stretching approach to mimic QP corrections, in particular for the Mn $d$ empty states. The latter, being only $1 \mathrm{eV}$ above the Fermi level, are essentially unaffected by the stretching approach.

We can thus conclude that at least the first peak in the Kerr rotation remains substantially unchanged for doping concentrations varying from $12.5 \%$ to $25 \%$, and it is barely affected by the details of the geometrical distribution of the Mn atoms.

As it appears from Eq. (5), a peak in the Kerr rotation can either have an "optical" origin, i.e., it stems from a vanishing value of the denominator, or a "magneto-optical" one, i.e., it 


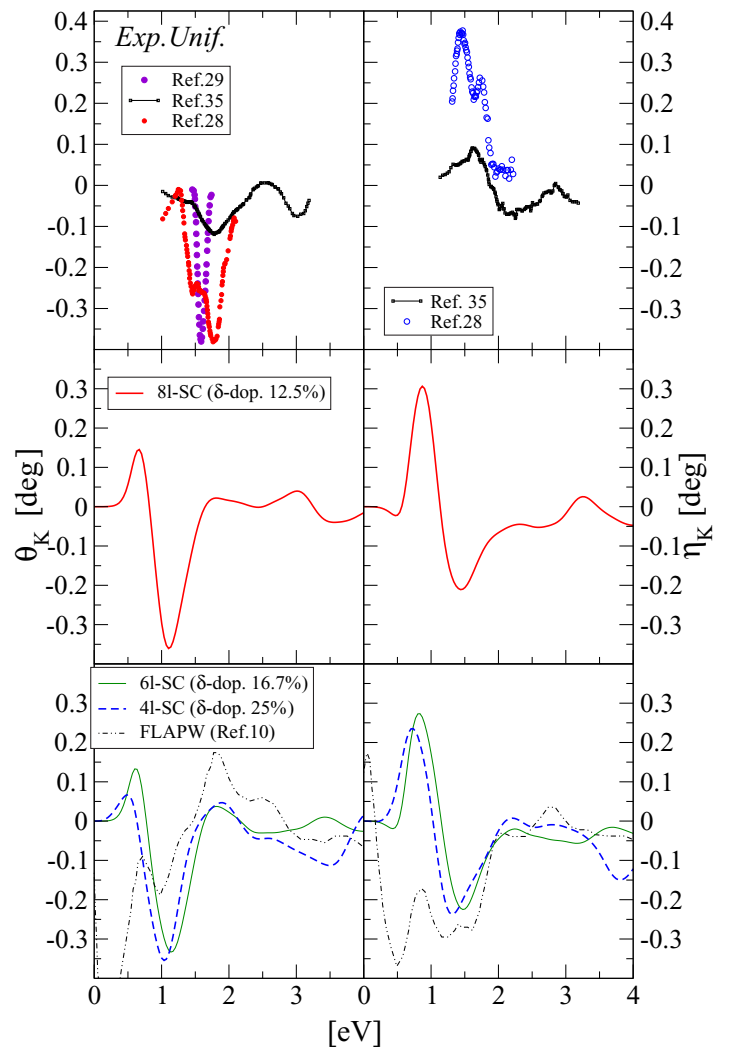

FIG. 9. (Color online) Kerr parameters computed at the IP-RPA level for three Mn $\delta$-doped GaAs heterostructures. Central panels show the spectrum of the $12.5 \% \mathrm{Mn} \mathrm{DFH}$ (red line); the results for the $16.7 \%$ and $25 \%$ Mn concentrations are shown in bottom panels (green and dashed blue lines, respectively). In the top panel, we show, for comparison, several experimental data for uniformly doped samples: black dots are for a 6\% Mn sample considered in Ref. [35], the red dots are for a $3 \% \mathrm{Mn}$ case (Ref. [28]), and violet dots refer to a $2 \% \mathrm{Mn}$ sample (Ref. [29]). Finally blue empty dots in right top panel are experimental data for the reflection MCD in a uniformly doped sample with 3\% Mn from Ref. [28]. In bottom panels, we report as dashed lines the results of FLAPW (LDA + stretched bands) calculations for a uniformly doped system, from Ref. [10].

arises from maximum/minimum of $\epsilon_{x y}(\omega)$ in the numerator. For the uniformly doped system the minimum close to $1 \mathrm{eV}$ has been reported to be of "optical" origin $[9,10]$.

To analyze whether this is the case also for the DFH, we plot in Fig. 10 the diagonal and the off-diagonal dielectric function. We clearly see that $\operatorname{Im}\left[\epsilon_{x y}\right]$ has a maximum at $1 \mathrm{eV}$ and also that both $\operatorname{Re}\left[\epsilon_{x x}\right]$ and $\operatorname{Im}\left[\epsilon_{x x}\right]$ are small in the same region. So the corresponding peaks of the Kerr rotation, in the present case, have both optical and magneto-optical origin.

Comparing the computed Kerr parameters with the computed DOS and its projections (see Figs. 2-5), we see that a key role is likely played by the empty state induced by the $\mathrm{Mn}$ doping, as discussed in Sec. II (see also Fig. 6). Indeed we note that this state is located about $1 \mathrm{eV}$ above the Fermi level. Thus the maximum (in absolute value) of the rotation is likely due to transitions from the valence band to this state, i.e., it is due to a transition involving both $d$ and $s p^{3}$ states.

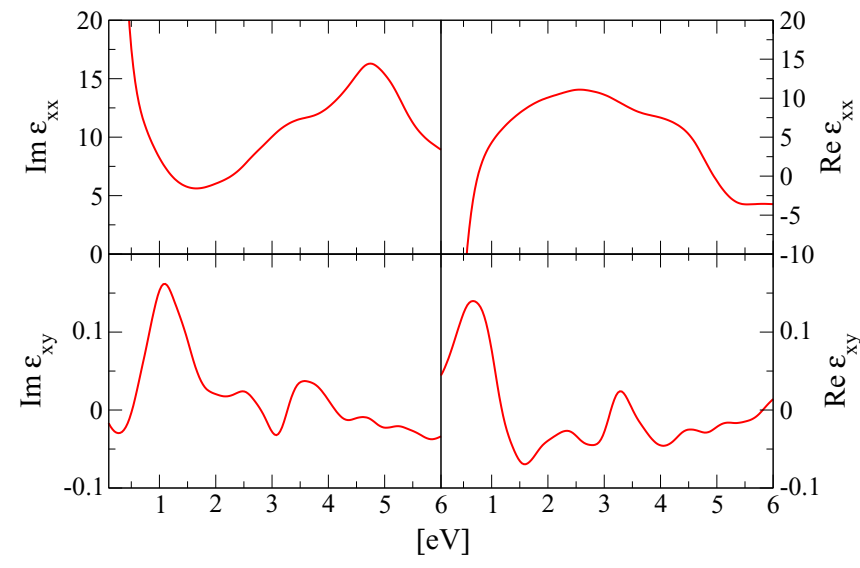

FIG. 10. (Color online) Real and imaginary part of the diagonal and off-diagonal components of $\epsilon$ for the Mn $\delta$-doped GaAs heterostructure corresponding to a $12.5 \%$ doping (Ref. [28]).

We thus suggest that in DFHs the MOKE could be substantially affected by electron/hole doping. Indeed, varying the hole concentration, the semimetallic character of the system can be substantially affected, leading to a very different absorption spectra, in particular in the region close to or below $1 \mathrm{eV}$ as already suggested for the uniformly doped system [30]. This conclusion is also supported by previous all-electron results for the uniform-doping case, where the Kerr spectrum is shown to change significantly when As antisite defects are considered together with Mn doping [9].

\section{B. Faraday effect and transmission magneto-optical dichroism}

While the Kerr effect and reflection MCD imply measuring the light reflected by a magnetic material, the corresponding effect for light transmitted through a magnetic medium is known as the Faraday effect. In polar geometry (the one considered in the present study), when a linear polarized light propagates parallel to the magnetization of a ferromagnet, a Faraday rotation is observed as a rotation of the polarization plane of the transmitted light $\left(\theta_{F}\right)$. In terms of circularly polarized reflective indexes, the Faraday rotation $\theta_{F}$ and the Faraday ellipticity $\eta_{F}$ read [6]

$$
\theta_{F}(\omega)+i \eta_{F}(\omega)=i \frac{\omega}{2 c}\left[n_{+}(\omega)-n_{-}(\omega)\right] L,
$$

where $L$ is the sample thickness and $c$ the speed of light. We evaluated the Faraday parameters per unit length for the Mn $\delta$-doped GaAs in a similar way as done for Kerr parameters; our results are displayed in Fig. 11. As the Kerr parameters are related to the R-MCD, the Faraday parameters are related to the transmission MCD [31] (T-MCD):

$$
T_{\mathrm{MCD}}(\omega)=\frac{T_{+}-T_{-}}{T_{+}+T_{-}}
$$

which, at normal incidence, is proportional to $\Delta \alpha(\omega)$, i.e., the difference of the absorption coefficient $\alpha_{ \pm}(\omega)=2 \omega \operatorname{Im}\left[n_{ \pm}(\omega)\right] / c$ between the right and the left circularly polarized light:

$$
\Delta \alpha(\omega)=\alpha_{+}(\omega)-\alpha_{-}(\omega)=-\frac{4 \eta_{F}(\omega)}{L} .
$$




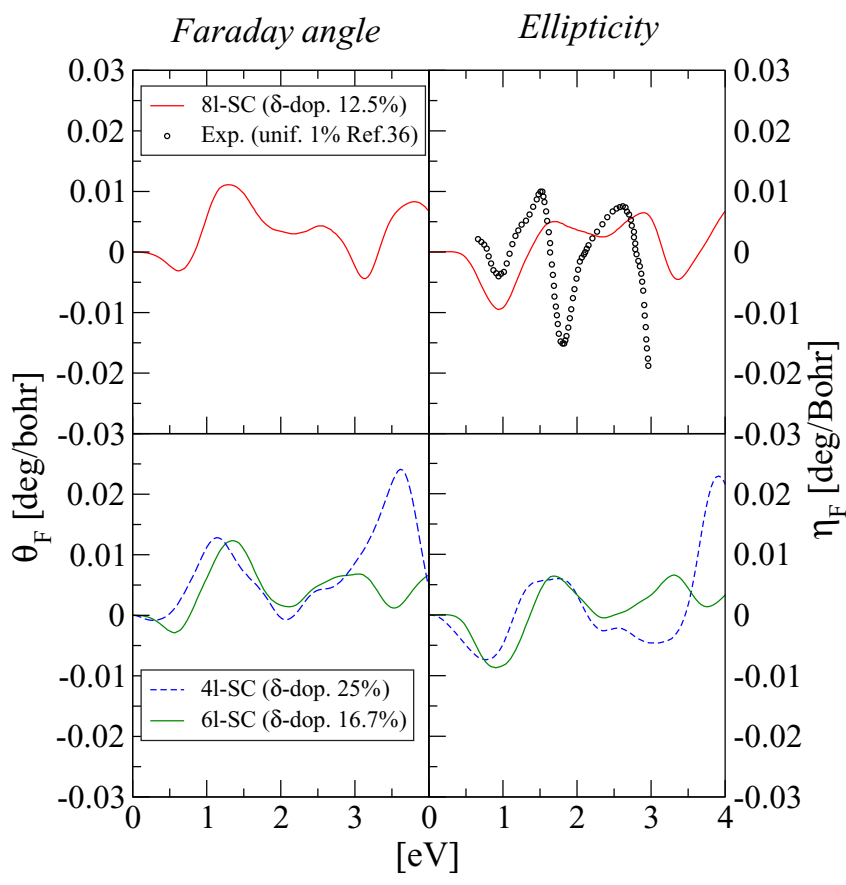

FIG. 11. (Color online) Faraday parameters/transmission MCD computed at the IP-RPA level for three $\mathrm{Mn} \delta$-doped $\mathrm{GaAs}$ heterostructures, corresponding to $12.5 \%, 16.7 \%$, and $25 \%$ Mn concentrations. Black empty dots are experimental data for uniformly doped samples with $1 \% \mathrm{Mn}$, from Ref. [36].

Thus we can compare the results of our simulations with T-MCD experimental data for diluted concentration [32] in randomly Mn-doped GaAs.

The first thing that we observe is that, despite that the physics of the Faraday effect and T-MCD is very similar to that of the Kerr effect and R-MCD, in this case the results are slightly more sensitive to the doping concentration. At least the region above $2 \mathrm{eV}$ is affected going from $12.5 \%$ to $25 \%$ doping. However, as in the case of the Kerr effect, there is a structure slightly above $1 \mathrm{eV}$ which is substantially unchanged varying the doping concentration.

To investigate possible applications of DFHs as Faraday rotators, in the following we compare some of our computed optical and magneto-optical data with the corresponding quantities of bulk transition metals, which are typical textbook examples for the Faraday effect [33].

We consider the 4-layer DFH and the peak at $\approx 1 \mathrm{eV}$ : according to our simulation, the Faraday rotation is $\theta_{F} \simeq 60^{\circ} \mu \mathrm{m}^{-1}$, significantly larger than the typical values for $\mathrm{Fe}, \mathrm{Co}$, and $\mathrm{Ni}$ $\left(35^{\circ}, 36^{\circ}, 10^{\circ} \mu \mathrm{m}^{-1}\right.$, respectively) [33] (taking into account the $0.6 \mathrm{eV}$ redshift of our computed spectra with respect to the experimental ones, these values are taken at $\lambda=820 \mathrm{~nm}$, i.e., at about $1.5 \mathrm{eV}$ ). On the other hand, DFHs have a lower optical absorption coefficient, which is desirable for efficient transmission. The optical penetration depth, defined as the length over which an electromagnetic wave of given frequency encounters a $1 / e$ reduction of its intensity-proportional to the inverse of the absorption coefficient-is about $36 \mathrm{~nm}$ for our 4-layer DFH. For comparison, the optical penetration depth of transition metals is of the order of $15 \mathrm{~nm}$.

\section{CONCLUSIONS}

In conclusion, our results for $\mathrm{Mn} \delta$-doped GaAs show that such digital ferromagnetic heterostructures have optical absorption and magneto-optical spectra displaying both similarities and differences with respect to previously studied uniformly doped MnGaAs systems. The 50\% Mn doping case (one layer of GaAs between MnAs layers) corresponds to a system with qualitatively different properties, having lost the half-metallicity. All other structures, corresponding to 12\%-25\% Mn doping, have optical absorption spectra which change gradually with the Mn concentration. The resulting spectra compare well with the available experimental data for the low-doping $(\leqslant 12 \%)$ system and theoretical data for the higher doping (25\%), for the uniformly doped case. With increasing Mn concentration the typical bulk GaAs spectral shape becomes less and less structured. In agreement with the experimental findings the peak at $\simeq 3 \mathrm{eV}$ is strongly suppressed, likely because of the hole doping induced by the Mn impurities, which screens the electron-hole interaction responsible for the creation of the excitonic peak. The peak at $\simeq 5 \mathrm{eV}$ is instead less affected.

Although the absorption spectra of the $\delta$-doped materials share the essential features of those of uniformly Mn-doped GaAs at similar concentrations, the effect of local fields is enhanced, due to the stronger nonuniformity of the $\delta$-doped system. This effect is particularly evident when the light polarization has a large component along the growth direction, while it is less important when the polarization lays in the plane of the Mn layers. MOKE spectra do not show a clear trend with the doping concentration: essentially, the maximum value of Kerr rotation is constant, and quite similar to that of the uniformly doped material $[9,10]$. Although the Faraday ellipticity is more sensitive to the doping concentration, also in this case the low-energy spectrum remains substantially unchanged moving from the high-doping case (25\%) to lower doping. On the other hand, the important role of the hole shared between the Mn $d$ and GaAs $s p^{3}$ states suggests that the studied DFHs could be very sensitive to a further doping with electrons.

\section{ACKNOWLEDGMENTS}

We acknowledge the CINECA and the Regione Lombardia award under the LISA initiative, for the availability of highperformance computing resources and support, the Fondazione Cariplo (Italy) for funding (Grant No. 2009-2552) within the project OSEA (Oxides for Spin Electronic Applications), and R. Colnaghi for technical support. One of us (G.O.) acknowledges the ETSF-Italy [34] for support.
[1] K. Sato, L. Bergqvist, J. Kudrnovský, P. H. Dederichs, O. Eriksson, I. Turek, B. Sanyal, G. Bouzerar, H. Katayama-
Yoshida, V. A. Dinh, T. Fukushima, H. Kizaki, and R. Zeller, Rev. Mod. Phys. 82, 1633 (2010). 
[2] A. M. Nazmul, T. Amemiya, Y. Shuto, S. Sugahara, and M. Tanaka, Phys. Rev. Lett. 95, 017201 (2005).

[3] X. Chen, M. Na, M. Cheon, S. Wang, H. Luo, B. D. McCombe, X. Liu, Y. Sasaki, T. Wojtowicz, J. K. Furdyna, S. J. Potashnik, and P. Schiffer, Appl. Phys. Lett. 81, 511 (2002).

[4] S. A. Wolf, D. D. Awschalom, R. A. Buhrman, J. M. Daughton, S. von Molnr, M. L. Roukes, A. Y. Chtchelkanova, and D. M. Treger, Science 294, 1488 (2001).

[5] B. Behin-Aein, D. Datta, S. Salahuddin, and S. Datta, Nat. Nanotechnol. 5, 266 (2010).

[6] See, e.g., K. Shinagawa, in Magneto-Optics, edited by S. Sugano and N. Kojima, Springer Series in Solid State Sciences, Vol. 128 (Springer-Verlag, Heidelberg, 2000).

[7] R. K. Kawakami, E. Johnston-Halperin, L. F. Chen, M. Hanson, N. Guébels, J. S. Speck, A. C. Gossard, and D. D. Awschalom, Appl. Phys. Lett. 77, 2379 (2000).

[8] K. S. Burch, E. J. Singley, J. Stephens, R. K. Kawakami, D. D. Awschalom, and D. N. Basov, Phys. Rev. B 71, 125340 (2005).

[9] S. Picozzi, A. Continenza, M. Kim, and A. J. Freeman, Phys. Rev. B 73, 235207 (2006).

[10] A. Stroppa, S. Picozzi, A. Continenza, M. Y. Kim, and A. J. Freeman, Phys. Rev. B 77, 035208 (2008).

[11] M. Ogura and H. Akai, Phys. Rev. B 82, 184426 (2010).

[12] G. Onida, L. Reining, and A. Rubio, Rev. Mod. Phys. 74, 601 (2002).

[13] D. Sangalli, A. Marini, and A. Debernardi, Phys. Rev. B 86, 125139 (2012).

[14] P. Hohenberg and W. Kohn, Phys. Rev. 136, B864 (1964).

[15] W. Kohn and L. J. Sham, Phys. Rev. 140, A1133 (1965).

[16] J. P. Perdew and A. Zunger, Phys. Rev. B 23, 5048 (1981).

[17] P. Giannozzi, S. Baroni, N. Bonini, M. Calandra, R. Car, C. Cavazzoni, D. Ceresoli, G. L. Chiarotti, M. Cococcioni, I. Dabo, A. Dal Corso, S. Fabris, G. Fratesi, S. de Gironcoli, R. Gebauer, U. Gerstmann, C. Gougoussis, A. Kokalj, M. Lazzeri, L. Martin-Samos, N. Marzari, F. Mauri, R. Mazzarello, S. Paolini, A. Pasquarello, L. Paulatto, C. Sbraccia, S. Scandolo, G. Sclauzero, A. P. Seitsonen, A. Smogunov, P. Umari, and R. M. Wentzcovitch, J. Phys.: Condens. Matter 21, 395502 (2009).

[18] The total residual force is below $1.7 \mathrm{mRy} / \mathrm{bohr}$.

[19] H. J. Monkhorst and J. D. Pack, Phys. Rev. B 13, 5188 (1976).
[20] The density of states has been computed projecting the wave functions either on the $+S_{z}$ or the $-S_{z}$ spin channel. In this way we have been able extract the spin-resolved DOS from our calculations.

[21] G. Strinati, Riv. Nuovo Cimento Soc. Ital. Fis. 11, 1 (1988).

[22] A. Marini, C. Hogan, M. Grüning, and D. Varsano, Comput. Phys. Commun. 180, 1392 (2009).

[23] D. Kammerlander, S. Botti, M. A. L. Marques, A. Marini, and C. Attaccalite, Phys. Rev. B 86, 125203 (2012).

[24] L. X. Benedict, E. L. Shirley, and R. B. Bohn, Phys. Rev. B 57, R9385 (1998); G. Adragna, R. Del Sole, and A. Marini, ibid. 68, 165108 (2003).

[25] E. J. Singley, R. Kawakami, D. D. Awschalom, and D. N. Basov, Phys. Rev. Lett. 89, 097203 (2002); E. J. Singley, K. S. Burch, R. Kawakami, J. Stephens, D. D. Awschalom, and D. N. Basov, Phys. Rev. B 68, 165204 (2003).

[26] K. S. Burch, J. Stephens, R. K. Kawakami, D. D. Awschalom, and D. N. Basov, Phys. Rev. B 70, 205208 (2004).

[27] D. E. Aspnes and A. A. Studna, Phys. Rev. B 27, 985 (1983).

[28] R. Lang, A. Winter, H. Pascher, H. Krenn, X. Liu, and J. K. Furdyna, Phys. Rev. B 72, 024430 (2005).

[29] A. V. Kimel, G. V. Astakhov, A. Kirilyuk, G. M. Schott, G. Karczewski, W. Ossau, G. Schmidt, L. W. Molenkamp, and Th. Rasing, Phys. Rev. Lett. 94, 227203 (2005).

[30] E. M. Hankiewicz, T. Jungwirth, T. Dietl, C. Timm, and J. Sinova, Phys. Rev. B 70, 245211 (2004).

[31] M. Berciu, R. Chakarvorty, Y. Y. Zhou, M. T. Alam, K. Traudt, R. Jakiela, A. Barcz, T. Wojtowicz, X. Liu, J. K. Furdyna, and M. Dobrowolska, Phys. Rev. Lett. 102, 247202 (2009).

[32] K. Ando, T. Hayashi, M. Tanaka, and A. Twardowski, J. Appl. Phys. 83, 6548 (1998).

[33] See, e.g., J. M. D. Coey, Magnetism and Magnetic Materials (Cambridge University Press, Cambridge, 2010).

[34] A. Matsuura, N. Thrupp, X. Gonze, Y. Pouillon, G. Bruant, and G. Onida, Comput. Sci. Eng. 14, 22 (2012).

[35] E. Kojima, R. Shimano, Y. Hashimoto, S. Katsumoto, Y. Iye, and M. Kuwata-Gonokami, Phys. Rev. B 68, 193203 (2003).

[36] K. Ando, H. Saito, K. C. Agarwal, M. C. Debnath, and V. Zayets, Phys. Rev. Lett. 100, 067204 (2008). 